\title{
Attenuated Bordetella pertussis vaccine strain BPZE1 modulates allergen-induced immunity and prevents allergic pulmonary pathology in a murine model
}

\author{
H. Kavanagh ${ }^{1}$, C. Noone ${ }^{1}$, E. Cahill ${ }^{1}$, K. English ${ }^{1}$, C. Locht ${ }^{2,3}$ and B. P. Mahon ${ }^{1}$ \\ ${ }^{1}$ Cellular Immunology Laboratory, Institute of Immunology, National University of Ireland Maynooth, Maynooth, County Kildare, Ireland, ${ }^{2}$ Institut Pasteur de Lille, Lille, \\ France and ${ }^{3}$ Inserm U629, Institut Pasteur de Lille, Lille, France
}

Correspondence:

Bernard P. Mahon, Cellular Immunology Laboratory, Institute of Immunology,

National University of Ireland

Maynooth, Maynooth, County Kildare, Ireland.

E-mail:bp.mahon@nuim.ie

Cite this as: H. Kavanagh, C. Noone,

E. Cahill, K. English, C. Locht and

B. P. Mahon, Clinical \&t Experimental

Allergy, 2010 (40) 933-941.

\begin{abstract}
Summary
Background Virulent Bordetella pertussis, the causative agent of whooping cough, exacerbates allergic airway inflammation in a murine model of ovalbumin (OVA) sensitization. A live genetically attenuated $B$. pertussis mucosal vaccine, BPZE1, has been developed that evokes full protection against virulent challenge in mice but the effect of this attenuated strain on the development of allergic responses is unknown.

Objective To assess the influence of attenuated B. pertussis BPZE1 on OVA priming in a murine model of allergic airway inflammation.

Methods Mice were challenged with virulent or attenuated strains of B. pertussis, and sensitized to allergen (OVA) at the peak of bacterial carriage. Subsequently, airway

pathology, local inflammation and OVA-specific immunity were examined.

Results In contrast to virulent $B$. pertussis, live BPZE1 did not exacerbate but reduced the airway pathology associated with allergen sensitization. BPZE1 immunization before allergen sensitization did not have an adjuvant effect on allergen specific IgE but resulted in a

statistically significant decrease in airway inflammation in tissue and bronchoalveolar lavage fluid. BPZE1 significantly reduced the levels of OVA-driven IL-4, IL-5 and IL-13 but induced a significant increase in IFN- $\gamma$ in response to OVA re-stimulation.

Conclusions These data demonstrate that, unlike virulent strains, the candidate attenuated $B$. pertussis vaccine BPZE1 does not exacerbate allergen-driven airway pathology. BPZE1 may represent an attractive T-helper type 1 promoting vaccine candidate for eradication of whooping cough that is unlikely to promote atopic disease.
\end{abstract}

Keywords asthma, Bordetella pertussis, vaccine, whooping cough

Submitted 15 June 2009 and accepted 7 December 2009

\section{Introduction}

The pathogenesis of allergic asthma remains unclear. However, the current understanding involves the expansion of $\mathrm{CD}^{+}{ }^{+} \mathrm{T}$-helper type 2 (Th2) cells and a breakdown in tolerance to otherwise innocuous environmental allergens [1]. Genetic predisposition, coupled with environmental influences, appear to affect the regular suppression of Th2-mediated responses [2]. It has been hypothesized that abnormalities in the maturation of the lung during fetal and neonatal development may render the airways more susceptible to environmental allergens [3], favouring polarization towards the Th2 phenotype and thus, predisposing the individual to atopy and asthma. Allergen-driven production of IL-4, IL-5 and IL-13 are typical of allergic pathologies, and the secretion of such Th2-cytokines initiates isotype class switching of B cells towards IgE [4], increased mucus production [5] and recruitment of eosinophils to the airways $[6,7]$. Because $\mathrm{CD}^{+}{ }^{+} \mathrm{Th} 2$ cells represent a co-ordinating cell type in some allergies, it was suggested that the induction of counterbalancing responses might prevent the subsequent development of atopic disease [1]. According to this modification of Strachan's hygiene hypothesis [8], microbial exposure activates innate immune pathways that alter Th1, Th2 and Treg responses [1]. This results in the 
suppression of Th2 cell expansion, and a consequent inhibition of isotype switching to IgE. However, several studies have suggested that viral and bacterial infections play a role in exacerbation of respiratory disease. For example, respiratory syncytial virus $[9,10]$ and virulent Bordetella pertussis infection exacerbate allergic inflammation [11]. The latter has been shown to exacerbate airway pathology $[12,13]$ and stimulate IgE responses in a murine model of allergen-driven inflammation despite induction of Th1 immunity $[14,15]$. Th2-inducing acellular pertussis $(\mathrm{Pa})$ vaccines protect against $B$. pertussis-induced exacerbation of allergic asthma, but induce IL-13 both at a systemic and local level [16]. On the other hand, systemic immunization with Th1-inducing whole-cell pertussis (Pw) vaccines inhibits allergic airway responsiveness [17] indicating that protection from allergen-driven pathology is not simply modulation of Th1/Th2 responses, but may also be associated with the degree of airway damage at the time of priming, such that allergen priming in the mucosae during breakdown of the airway epithelial mesenchymal unit may be an equally important factor.

Recently, a genetically attenuated B. pertussis strain, BPZE1, has been developed as a live neonatal mucosal vaccine candidate against whooping cough [18]. This strain induces strong local and systemic immune responses upon a single intranasal delivery [18]. Administration via the nasal route mimics natural infection and is expected to promote long-lasting immunity in children from $<1$ month of age [19]. Although full attenuation of BPZE1 has been extensively documented in murine models, the influence of BPZE1 administration on allergen priming and allergen-induced pathology is not known, but is of obvious importance in ascertaining the safety of this live vaccine candidate.

This preclinical study compared a virulent pertussis strain, with the candidate vaccine, BPZE1, and examined their effect on the induction of ovalbumin (OVA)-induced airway inflammation using previously characterized animal models. We show here that, unlike virulent strains, attenuated BPZE1 did not exacerbate but reduced allergen-driven pathology.

\section{Materials}

Immunization, sensitization and airway delivery of ovalbumin and Bordetella pertussis

Eight- to 12-week-old, female BALB/c mice (Harlan, Oxon, UK) were used and maintained according to the regulations and guidelines of the Irish Department of Health, and the Research Ethics Committee of the National University of Ireland, Maynooth. Mice were exposed to live virulent or attenuated bacteria, and sensitized to allergen during infection. Virulent B. pertussis BPSM [20] and attenuated BPZE1 were cultured as previously described [21]. Attenuated or virulent strains at mid-log growth were administered to mice by aerosol using the respiratory challenge model and standard protocols [16]. Briefly mice received set time aerosol exposure which models natural mode of infection to achieve a colonizing dose of $1 \times 10^{6} \mathrm{CFU} /$ mouse. This dose was chosen for three reasons, it was the optimized dose from earlier studies [21], to be consistent with previous reports in this model $[16,17]$ and because this standard version of the respiratory challenge protocol had been used in assessment of clinical lots of previous $B$. pertussis vaccines [13]. At the peak of infection (10 days) and at 24 days, mice were sensitized by intraperitoneal injection $(0.3 \mathrm{~mL})$ of $100 \mu \mathrm{g} / \mathrm{mL}$ OVA in adjuvant (AlumImject ${ }^{\mathrm{TM}}$, Pierce, IL, USA). Mice were challenged intranasally $(30 \mu \mathrm{L})$ with OVA $(50 \mu \mathrm{g} / \mathrm{mL})$ on days $24,35,36$ and 37. Various control groups $(n=10)$ received sham delivery of sterile phosphate-buffered saline (PBS) in place of the active agent as previously described [12].

\section{Bronchoalveolar lavage and respiratory tract histology}

On day 37, mice were killed by lethal injection of sodium pentobarbital, and bronchoalveolar lavage fluid (BALF) was collected [12]. Total leucocytes and differential cell counts were performed as described [12], using Diff Quik/ Rapi-Diff II ${ }^{\mathrm{TM}}$ (Triangle Biomedical Sciences, Durham, NC, USA). Lungs from non-lavaged mice were removed and fixed in $10 \%(\mathrm{v} / \mathrm{v})$ formalin/PBS, embedded in paraffin, sectioned and stained with haematoxylin/eosin, or combined Discombes/Alcian blue and periodic acid-Schiff (PAS) stain [12]. Histopathological changes were graded according to an established semi-quantitative scoring system as mild, moderate or severe [16], and are shown for convenience in supporting information (Fig. S1).

\section{$T$ cell proliferation assay}

Splenocytes from mice were prepared as previously described [22] and incubated for $72 \mathrm{~h}$ with medium (negative control), OVA $(200 \mu \mathrm{g} / \mathrm{mL})$ or concanavalin A $(5 \mu \mathrm{g} / \mathrm{mL})$. Supernatants were removed after 48-h incubation for cytokine analysis, and cultures received fresh medium. Cells were incubated for the final $6 \mathrm{~h}$ with $\left[{ }^{3} \mathrm{H}\right]$-thymidine and proliferation was detected by liquid scintillation [23].

\section{Measurement of cytokines and antibody responses}

IL-5, IL-10, IL-13 and IFN- $\gamma$ from BALF and splenocyte supernatants were analysed by flow cytometry (BectonDickinson, Franklin Lakes, NJ, USA), using Cytometric Bead Array Flex Sets (BD Biosciences) according to the manufacturer's instructions. Standard curves and raw data were generated for each cytokine using FCAP Array v1.0.1 software (BD Biosciences). OVA-specific serum IgE was measured by ELISA as previously described [24] using a rat anti-mouse IgE monoclonal antibody (BD 
Pharmingen, San Diego, CA, USA). IgE concentration was expressed as $\mu \mathrm{g} / \mathrm{mL}$ after comparison with murine $\operatorname{IgE}$ standards (BD Pharmingen).

\section{Statistical analysis}

Values for all measurements were expressed as the mean \pm standard error of the mean. Statistical analysis was performed using GraphPad Prism ${ }^{\mathrm{TM}}$ software (GraphPad, San Diego, CA, USA). Comparison was made using the Kruskal-Wallis test, or the Mann-Whitney test as appropriate. Significance was denoted by $P$-value $<0.05$.

\section{Results}

Attenuated Bordetella pertussis BPZE1 prevents ovalbumin-driven allergic airway pathology

Virulent $B$. pertussis can exaggerate unrelated allergen priming in animal models [12] and has been associated with exacerbation of allergy in humans [11]. To assess the influence of the candidate vaccine on OVA-induced airway pathology, mice were challenged with either virulent BPSM, or attenuated BPZE1, and sensitized to OVA at the peak of bacterial carriage (a model previously shown to uncover the influence of infection on allergen-driven inflammation) $[12,13,16]$. In the absence of infection,
OVA-sensitized mice exhibited typical peribronchial and perivascular inflammation at day 38, which was not observed in control mice (Figs 1a and b). At this timepoint, pathology due to virulent bacterial infection alone has resolved [22]. Priming at the peak of virulent B. pertussis infection resulted in a more severe pathology when compared with non-infected sensitized mice, displaying strong perivascular inflammation and hypertrophied bronchiolar epithelium (Fig. 1c). In contrast, prior challenge with attenuated BPZE1 resulted in decreased peribronchial inflammation when compared with sensitized, BPSM-infected mice (Fig. 1d). Excessive mucus secretion from goblet cells is a characteristic feature of the allergic airway. An examination of lung tissues stained with PAS and Alcian blue demonstrated that prior immunization with BPZE1 in OVA-sensitized mice reduced mucus secretion when compared with those sensitized to OVA alone (Fig. 2). In contrast, virulent BPSM-infection exacerbated goblet cell hyperplasia and mucus secretion in OVA-sensitized mice (Fig. 2c). Consistent with these data were physiological observations of airway hyper-responsiveness (AHR) suggesting that prior immunization with BPZE1 resulted in significantly reduced bronchial hyperreactivity compared with mice primed while infected with virulent B. pertussis (supporting information Fig. S2). Taken together these data show that unlike infection with virulent $B$. pertussis, administration of attenuated
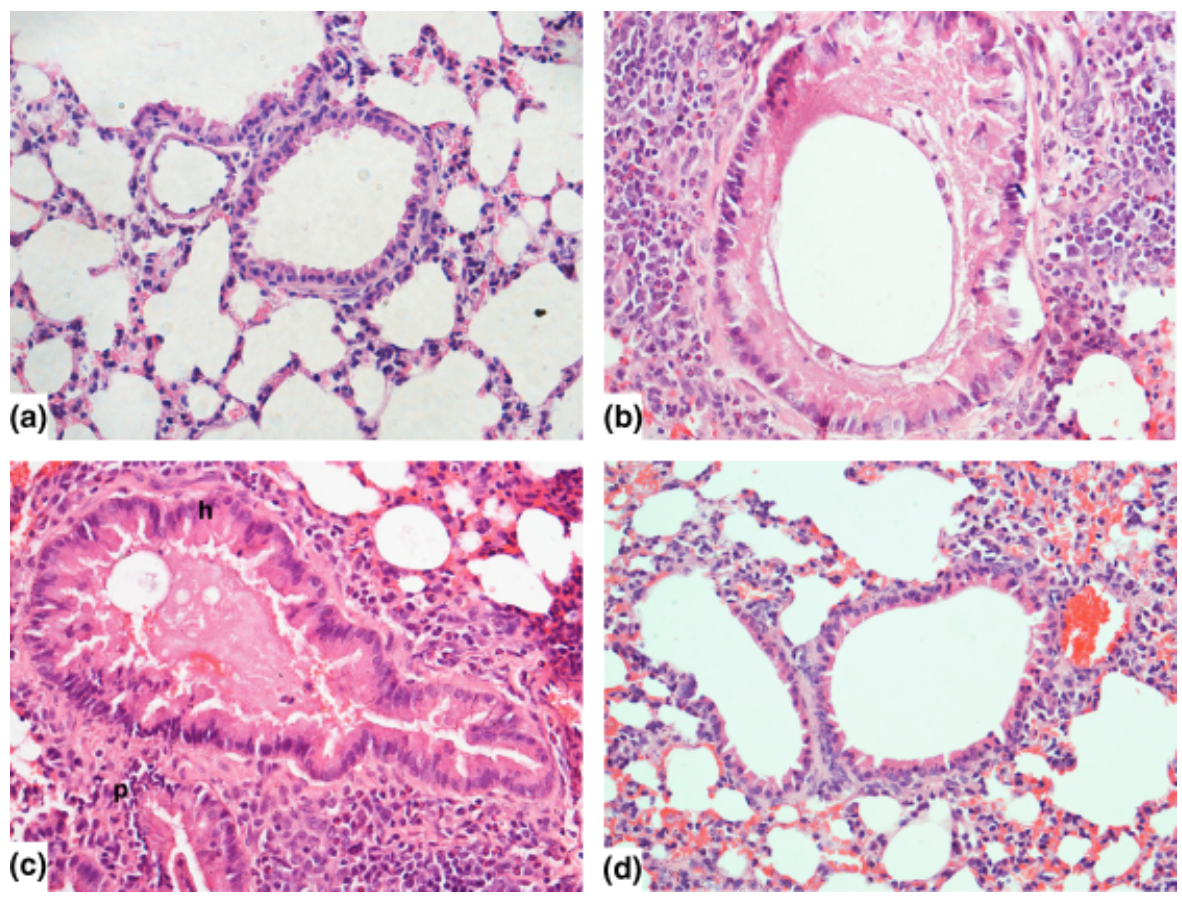

Fig. 1. Attenuated Bordetella pertussis BPZE1 reduces the severity of airway pathology induced by sensitizing allergen. Representative morphological changes at 38 days in bronchiolar transverse sections of lungs from (a) non-sensitized, (b) ovalbumin (OVA)-sensitized, (c) OVA-sensitized and infected with B. pertussis, (d) OVA-sensitized and immunized with BPZE1. Airway inflammation was detected using haematoxylin and eosin staining of fixed lung sections. $\mathrm{p}$ and $\mathrm{h}$ indicate perivascular inflammation and bronchiolar epithelial hypertrophy, respectively. All images are representative of multiple sections from five mice per experiment, repeated at least twice (i.e. $n>10$ ). Original magnification $\mathrm{a}-\mathrm{d} \times 400$. 

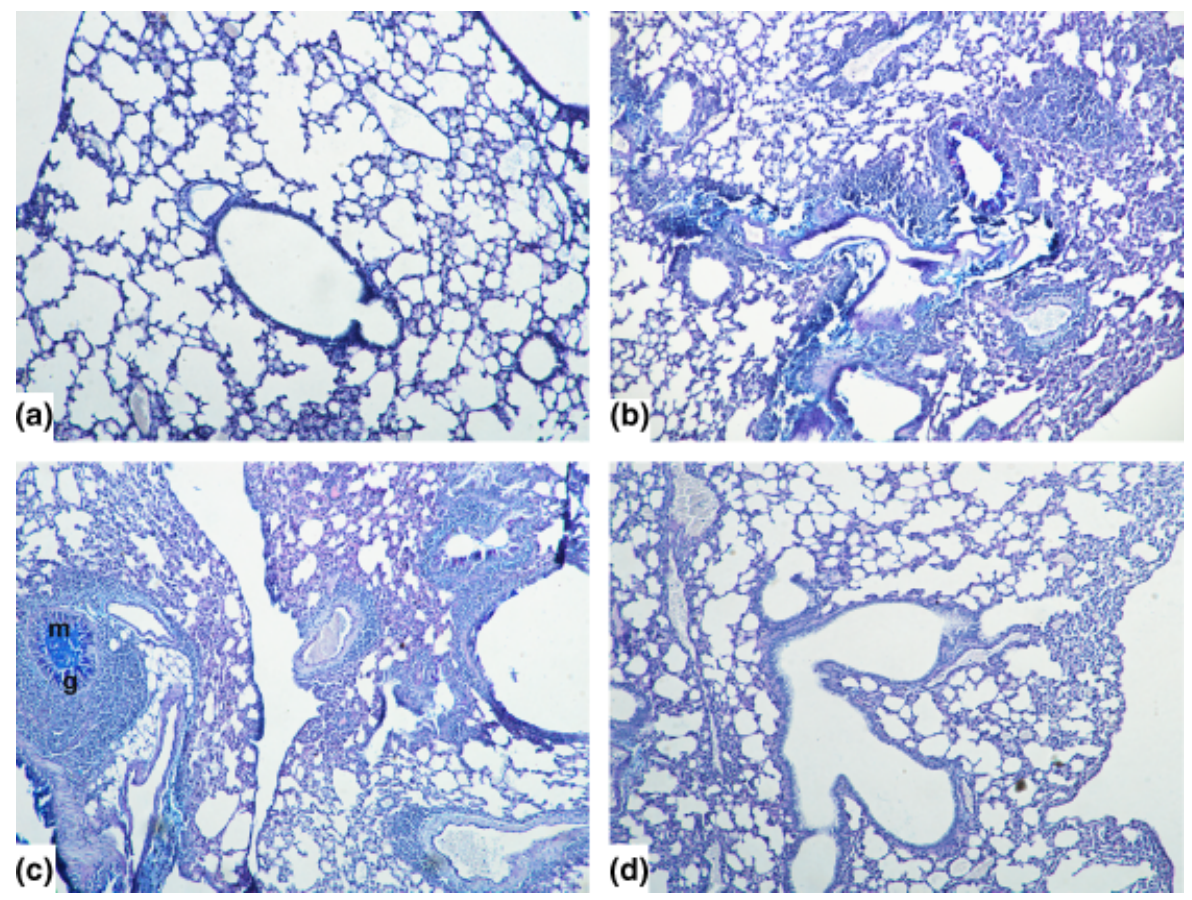

Fig. 2. Attenuated Bordetella pertussis BPZE1 reduces the severity of mucus hyperplasia to sensitizing allergen. Representative morphological changes at 38 days in transverse sections of bronchioles from (a) non-sensitized, (b) ovalbumin (OVA)-sensitized, (c) OVA-sensitized and infected with virulent B. pertussis, (d) OVA-sensitized and vaccinated with BPZE1. Airway inflammation was detected using combined Discombes/Alcian blue/ periodic acidSchiff staining on lung sections. g and m indicate goblet cell hyperplasia and mucus secretion, respectively. All images are representative of multiple sections from five mice per experiment repeated at least twice (i.e. $n>10$ ). Original magnification $\times 100$.

B. pertussis BPZE1 did not enhance, but reduced the pathology associated with allergen sensitization.

\section{Attenuated Bordetella pertussis BPZE1 prevents ovalbumin-driven allergic airway inflammation}

The capacity for live attenuated B. pertussis BPZE1 to influence OVA-induced inflammatory influx to the respiratory tract was examined. OVA sensitization resulted in characteristic inflammation with eosinophils, neutrophils, macrophages and lymphocytes detected in BALF not seen in control mice (Fig. 3). Mice sensitized during virulent $B$. pertussis infection showed a similar pattern of inflammation. However, in marked contrast, immunization with live attenuated BPZE1 before OVA sensitization resulted in significantly reduced inflammatory infiltration of the airways $(P<0.05)$ for all cells examined. Thus a major finding of this study was that attenuated B. pertussis BPZE1 reduced OVA-driven allergic airway inflammation typically seen in this model.

Bordetella pertussis BPZE1 does not enhance serum immunoglobulin $E$ responses to sensitizing allergen

$B$. pertussis is a known adjuvant for IgE, as is active pertussis toxin $[14,15]$. Therefore the influence of BPZE1 on allergic sensitization was examined by measuring the concentration of OVA-specific IgE in serum in these mice. Unlike non-sensitized controls, OVA sensitization induced significant levels of IgE as expected (Fig. 4). Allergenspecific IgE responses in mice exposed to attenuated BPZE1 before OVA sensitization were not enhanced but were not significantly different to those receiving OVA alone. However, compared with mice infected with virulent BPSM in combination with OVA sensitization, attenuated BPZE1 immunization resulted in a significant reduction $(P<0.05)$ of OVA-induced IgE (Fig. 4). Therefore, unlike virulent $B$. pertussis BPSM which damages the airways, live attenuated B. pertussis BPZE1 delivered before allergen priming did not enhance or have an adjuvant effect upon the IgE response to allergen.

\section{Bordetella pertussis BPZE1 modulates recall cytokine responses to sensitizing allergen}

Attenuated BPZE1 has a radically different effect on allergen-driven airway pathology to virulent $B$. pertussis. To further investigate this, the influence of bacterial exposure on the pattern of allergen-induced cellular immune responses was characterized. OVA-specific cytokine production in both spleen cell preparations and BALF was assessed in the groups described above, in order to evaluate the influence of BPZE1 on allergen-induced priming. As expected, OVA sensitization alone induced 

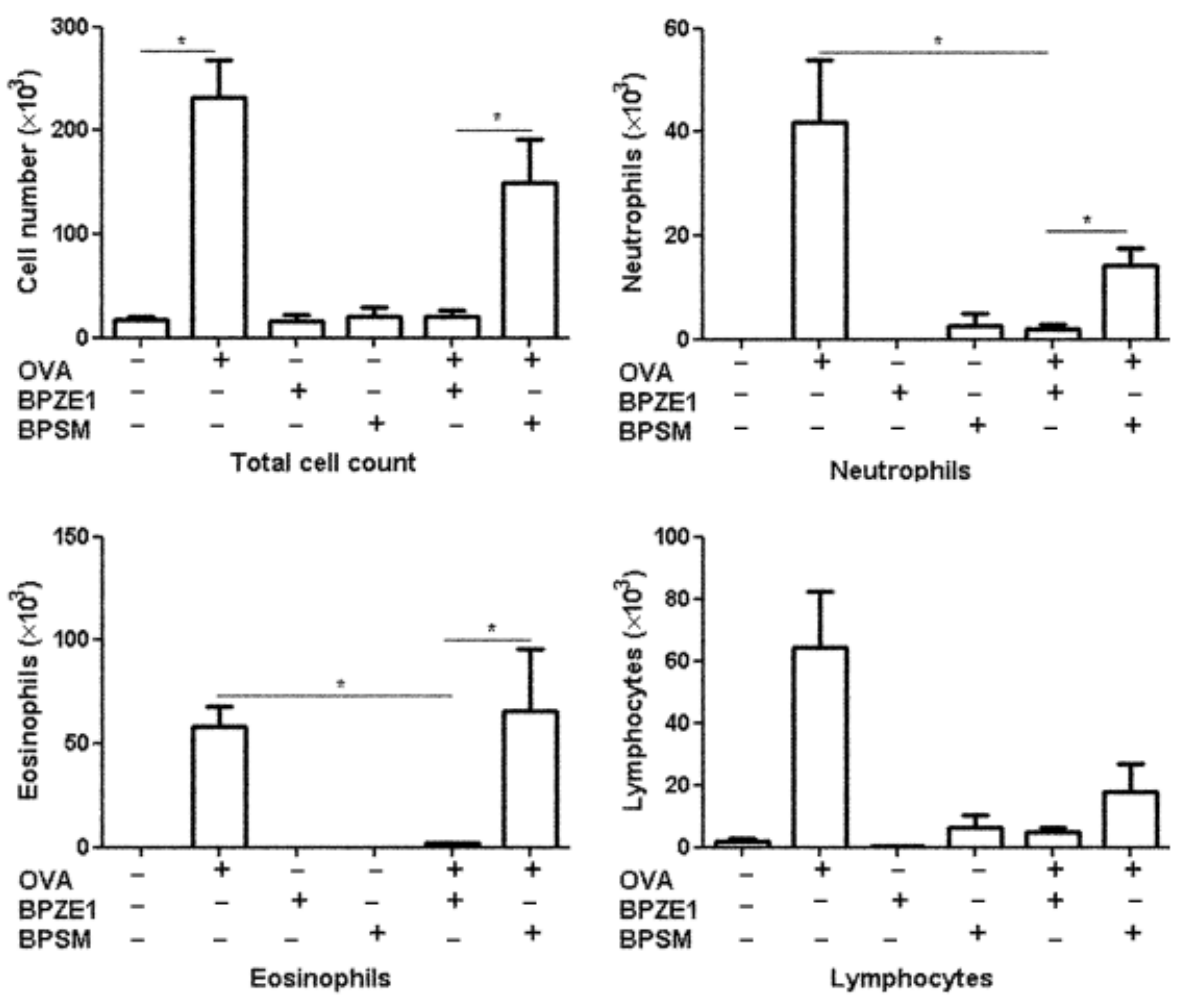

Fig. 3. Attenuated Bordetella pertussis BPZE1 reduces the cell infiltrate of bronchoalveolar lavage fluid (BALF). Effect of virulent BPSM infection, attenuated BPZE1 challenge and/or ovalbumin (OVA) sensitization on BALF composition $24 \mathrm{~h}$ after final OVA exposure. Negative controls were sham infected/sensitized with saline. BALF was examined for the total cell number (a), or the presence of neutrophils (b), eosinophils (c) or lymphocytes (d). The data are representative of triplicate measurements from at least five animals and repeated twice. Results are expressed as mean \pm standard error of the mean of cell number. ${ }^{*} P<0.05$.

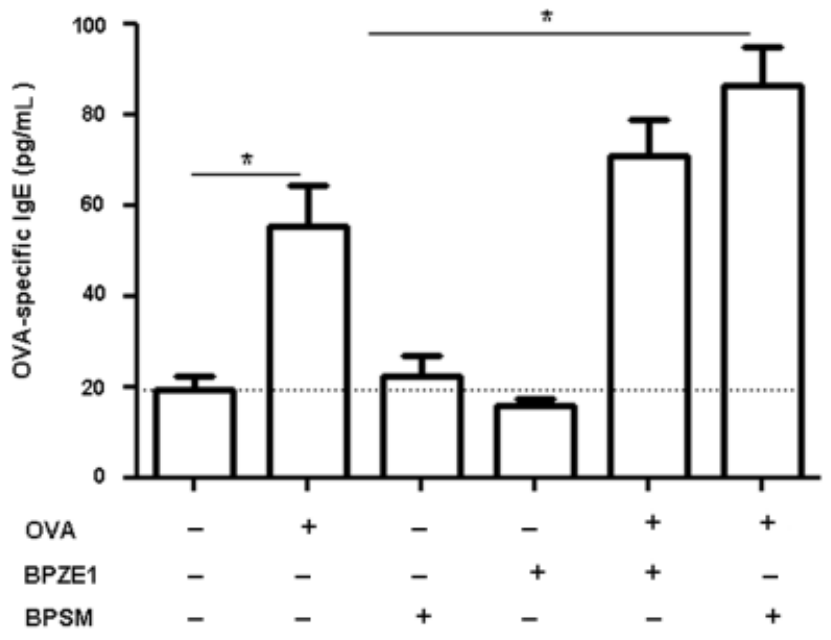

Fig. 4. Attenuated Bordetella pertussis BPZE1 reduces allergen-induced IgE. Ovalbumin (OVA)-specific IgE in serum elicited in response to OVA sensitization. Sera were collected on day 38 and OVA-specific serum IgE levels were measured by ELISA. The data presented are representative of two experiments; in each case, at least five animals were assessed, and each individual assessment was performed in triplicate. Concentrations below $100 \mathrm{pg} / \mathrm{mL}$ were considered negative. Results are expressed as mean antibody concentrations \pm standard error of the mean. ${ }^{*} P<0.05$. high levels of the Th2 cytokines IL-4, IL-5 and IL-13 (Fig. 5). Neither virulent BPSM nor attenuated BPZE1 alone induced any recall response to OVA, but both provoked strong Th1 responses to $B$. pertussis antigens (data not shown), as previously reported [16]. Virulent BPSM challenge before sensitization did not induce a significant reduction in OVA-specific IL-5 or IL-13 and did not increase IFN- $\gamma$ significantly (Fig. 5). However, a striking increase in IL-4 in BALF was observed when compared with those sensitized to OVA alone. In contrast, BPZE1 modulated the allergen response. Prior BPZE1 significantly reduced the levels of OVA-induced IL-5 $(P<0.005)$, IL-13 $(P<0.05)$ and IL-4 $(P<0.005)$. This was an immunomodulatory effect because suppression was not global; BPZE1 significantly increased IFN $-\gamma$ in BALF and from splenocytes re-stimulated with OVA $(P<0.05)$. In summary, BPZE1 did not promote Th2 cytokine induction to allergen, but rather redirected this to a Th1-like response.

\section{Discussion}

The present study combined murine infection and sensitization models to demonstrate that an attenuated strain of 

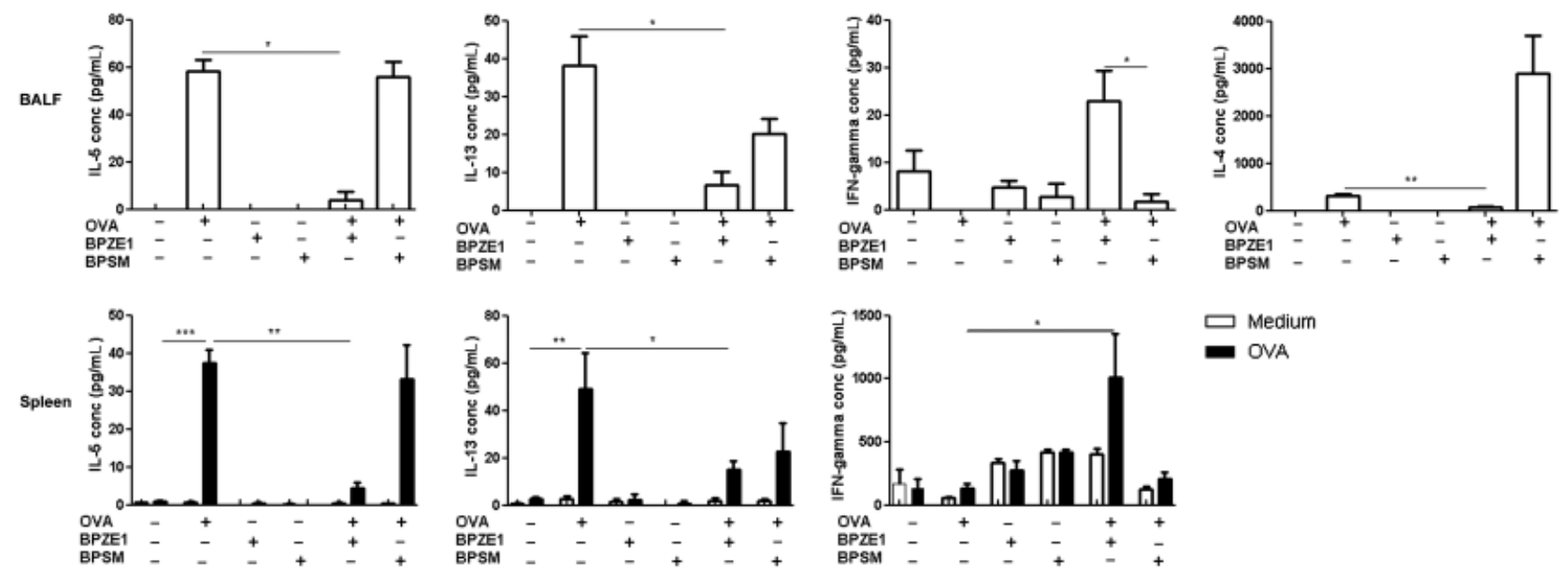

Fig. 5. Cell-mediated immune responses from splenocytes to ovalbumin (OVA) and in bronchoalveolar lavage fluid, elicited by OVA sensitization 10 days following prior exposure to attenuated (BPZE1) or virulent (BPSM) Bordetella pertussis infection. Negative symbols indicate sham sensitization or challenge with phosphate-buffered saline. Cytokine responses from similar cultures are shown for (a) IL-5, (b) IL-13, (c) IFN- $\gamma$. Responses are representative of duplicate experiments, each of which were determined independently from at least five mice per group in each case and are expressed as means \pm standard error of the mean. ${ }^{*} P<0.05,{ }^{* *} P<0.005,{ }^{* * *} P<0.0005$.

B. pertussis, BPZE1, did not enhance but reduced allergen-driven airway pathology. BPZE1 prevented OVA-driven inflammation of the airways, assessed by histological and qualitative analysis of mucus production. Furthermore, BPZE1 diminished the severity of allergendriven inflammation in the lungs. Eosinophilic and neutrophilic infiltration was significantly reduced in BPZE1-immunized mice sensitized to OVA. Virulent BPSM enhanced IL-4 in BALF, whereas BPZE1 modulated allergen responses towards a Th1 phenotype, demonstrated by a marked increase in IFN- $\gamma$. Likewise, analysis of recall responses to allergen showed that BPZE1 modulated responses away from IL- 5 and IL-13 towards IFN- $\gamma$. In contrast to virulent $B$. pertussis, BPZE1 demonstrated no adjuvant associated increase in allergen-specific IgE. Taken together, these data demonstrate that attenuated BPZE1 acts as a powerful immunomodulator that suppresses allergen-driven pathology at both a local and systemic level.

Some versions of the hygiene hypothesis suggest that Th1-inducing infections may have an inhibitory effect on the development of atopy [25]. However, previous studies have demonstrated that virulent $B$. pertussis enhanced the severity of airway pathology $[12,13]$ despite induction of Th1 immunity. In contrast, systemic immunization with a Th1-inducing $\mathrm{Pw}$ vaccine inhibited allergic airway responsiveness [13]. These seemingly counter-intuitive findings suggest that protection from allergen-driven pathology is linked not just to $\mathrm{CD}^{+}{ }^{+} \mathrm{T}$ cell profile, but also to the degree of airway damage at the time of priming [14]. This can be probed using virulent (airway damaging) and attenuated (non-damaging) bacterial strains. The present study supports those findings showing that prior immunization with BPZE1 that does not damage the airway epithelium [26] resulting in significantly reduced allergic pathology, compared with those mice sensitized while infected with virulent $B$. pertussis that induces airway damage [26]. Vaccination with $\mathrm{Pw}$ has been reported to protect against $B$. pertussis exacerbation of OVA-induced AHR in a murine model of allergic airway inflammation [17] and it has been shown that there is no allergy-promoting effect in response to common childhood vaccines, including pertussis vaccines [27]. However, much less is known about protective effects of childhood vaccines against atopy, others have shown that live attenuated vaccines, such as oral poliomyelitis vaccine or Bacillus-Calmette-Guérin, inhibited the development of asthma and allergic disease [28]. However, the mechanisms underlying the beneficial influence of attenuated BPZE1 on allergen-driven pathology may be multiple and inter-linked. It is known in other models that immune interaction between infection and allergen sensitization critically depends on timing of challenges [29]. In this model, the challenge schedule was chosen to examine the effect of prior immunization on allergen sensitization and for consistency with previous reports [12, 16, 17]. However, it will be important to examine the reverse scenario to assess the effect of immunization on preexisting allergen sensitivity, such as that seen using unmethylated $\mathrm{CpG}$ oligodeoxynucleotides which inhibited ragweed allergen induced lung inflammation in presensitized animals [30].

Pathology in the OVA model is driven by Th2 cytokines, in particular IL-5 and IL-13. However, there are two 
components to this; the first is the induction of $\operatorname{IgE}[31$, 32] and classical atopic inflammation. The second is Th2driven fibrosis/remodelling [33], which can be antagonized by IFN- $\gamma[34,35]$. The observations here that allergen-specific IgE was not significantly changed while pathology was reduced in mice immunized with BPZE1 before OVA sensitization (Figs 1 and 4) suggest that a mechanism of protection other than IgE reduction is involved here. The inhibition of allergic airway pathology might be explained by the modulation of the key cytokines involved in remodelling. This study demonstrated that BPZE1 shifted systemic immune responses to OVA away from IL-4, IL-5 and IL-13 towards IFN- $\gamma$. Both IL-4 and IL-5 contribute to tissue damage and remodelling due to their function as mediators of eosinophil recruitment $[36,37]$. Infection with virulent $B$. pertussis exaggerates the OVA-induced inflammatory influx to the respiratory tract, with an increase in eosinophils (Fig. 3c), and an associated increase in the severity of airway pathology (Fig. 1e). Conversely, administration of attenuated BPZE1 before allergen sensitization significantly reduced inflammatory infiltration (Fig. 3). Airway mucus hypersecretion is in part driven by IL-13 and is a major pathophysiological feature of both allergic asthma [38] and whooping cough [39]. Therefore, it is not surprising that mucus production mirrored IL-13 levels in this study and was significantly reduced in sensitized mice previously exposed to BPZE1 (Fig. 5c). Taken together, these data suggest that immune modulation in this model has its greatest impact on mucus secretion and remodelling rather than IgE induction. Since attenuation of BPZE1 is based on the genetic removal or attenuation of three major toxins, pertussis toxin, tracheal cytotoxin and dermonecrotic toxin [18], this study suggests that one, or a combination, of the attenuated virulence factors in BPZE1 plays a role in the adjuvant effect observed with virulent $B$. pertussis strains. The key finding here is that in contrast to virulent $B$. pertussis, attenuated BPZE1 did not exacerbate allergen-induced airway pathology in a murine model.

BPZE1 has been developed as a new candidate for use as a live, intranasal, single-dose neonatal vaccine against whooping cough [18]. Most current vaccination regimes require three doses, beginning at 2 months of age necessitating 6 months for optimal protection [40]. Therefore, there is a need for vaccines that induce strong protection against $B$. pertussis in neonates. The data presented here support the use of this candidate vaccine even for populations where exposure to allergens and atopy is prevalent. Allergic airway inflammation is not simply a balance between Th1 and Th2 responses [41]. It might be that the key beneficial feature of BPZE1 is the combination of a Th1 skewed response, combined with the absence of induced airway pathology. This is supported by previous reports in which exacerbation of airway pathology to allergen was associated with allergen priming during a period of airway damage or remodelling [12, 42, 43]. This combined benefit makes BPZE1 an attractive candidate as a neonatal vaccine against whooping cough.

\section{Acknowledgements}

This work is part of the CHILD-INNOVAC network (Collaborative Project) supported by the European Commission under the Health Cooperation Work Programme of the 7th Framework Programme (Grant agreement no. 201502). H. K. is funded by the Irish Health Research Board.

\section{References}

1 Romagnani S. Immunologic influences on allergy and the TH1/ TH2 balance. J Allergy Clin Immunol 2004; 113:395-400.

2 Postma DS, Bleecker BE, Amelung PJ et al. Genetic susceptibility to asthma-bronchial hyperresponsiveness coinherited with a major gene for atopy. N Engl J Med 1995; 333:894-900.

3 Holgate ST, Davies DE, Lackie PM, Wilson SJ, Puddicombe SM, Lordan JL. Epithelial-mesenchymal interactions in the pathogenesis of asthma. J Allergy Clin Immunol 2000; 105:193-204.

4 Del Prete G, Maggi E, Parronchi P et al. IL-4 is an essential factor for the IgE synthesis induced in vitro by human T cell clones and their supernatants. J Immunol 1988; 140:4193-8.

5 Wilder JA, Collie DD, Wilson BS, Bice DE, Lyons CR, Lipscomb MF. Dissociation of airway hyperresponsiveness from immunoglobulin E and airway eosinophilia in a murine model of allergic asthma. Am J Respir Cell Mol Biol 1999; 20:1326-34.

6 Schleimer RP, Sterbinsky SA, Kaiser J et al. IL-4 induces adherence of human eosinophils and basophils but not neutrophils to endothelium. Association with expression of VCAM-1. J Immunol 1992; 148:1086-92.

7 Walsh GM, Wardlaw AJ, Hartnell A, Sanderson CJ, Kay AB. IL-5 enhances the in vitro adhesion of human eosinophils, but not neutrophils, in a leucocyte integrin (CD11/18)-dependent manner. Immunology 1990; 71:258-65.

8 Romagnani S. Human TH1 and TH2 subsets: regulation of differentiation and role in protection and immunopathology. Int Arch Allergy Immunol 1992; 98:279-85.

9 Johnson TR, Graham BS. Secreted respiratory syncytial virus G glycoprotein induces interleukin-5 (IL-5), IL-13, and eosinophilia by an IL-4-independent mechanism. J Virol 1999; 73:8485-9.

10 Matsuse H, Behera AK, Kumar M, Rabb H, Lockey RF, Mohapatra SS. Recurrent respiratory syncytial virus infections in allergensensitized mice lead to persistent airway inflammation and hyperresponsiveness. J Immunol 2000; 164:6583-92.

11 Harju TH, Leinonen M, Nokso-Koivisto J et al. Pathogenic bacteria and viruses in induced sputum or pharyngeal secretions of adults with stable asthma. Thorax 2006; 61:579-84.

12 Ennis DP, Cassidy JP, Mahon BP. Prior Bordetella pertussis infection modulates allergen priming and the severity of airway pathology in a murine model of allergic asthma. Clin Exp Allergy 2004; 34:1488-97.

13 Mills KH, Brady M, Ryan E, Mahon BP. A respiratory challenge model for infection with Bordetella pertussis: application in the assessment of pertussis vaccine potency and in defining the 
mechanism of protective immunity. Dev Biol Stand 1998; 95:31-41.

14 Ryan EJ, Nilsson L, Kjellman N, Gothefors L, Mills KH. Booster immunization of children with an acellular pertussis vaccine enhances Th2 cytokine production and serum IgE responses against pertussis toxin but not against common allergens. Clin Exp Immunol 2000; 121:193-200.

15 Nilsson L, Grüber C, Granström M, Björkstén B, Kjellman NI. Pertussis IgE and atopic disease. Allergy 1998; 53:1195-201.

16 Ennis DP, Cassidy JP, Mahon BP. Acellular pertussis vaccine protects against exacerbation of allergic asthma due to Bordetella pertussis in a murine model. Clin Diagn Lab Immunol 2005; 12:409-17.

17 Ennis DP, Cassidy JP, Mahon BP. Whole cell pertussis vaccine protects against exacerbation of allergic asthma. Immunol Lett 2005; 97:91-100.

18 Mielcarek N, Debrie AS, Raze D et al. Live attenuated B. pertussis as a single-dose nasal vaccine against whooping cough. PLoS Pathog 2006; 2: 662-70.

19 Mascart F, Verscheure V, Malfroot A et al. Bordetella pertussis infection in 2-month-old infants promotes type $1 \mathrm{~T}$ cell responses. J Immunol 2003; 170:1504-9.

20 Menozzi FD, Mutombo R, Renauld G et al. Heparin-inhibitable lectin activity of the filamentous hemagglutinin adhesin of Bordetella pertussis. Infect Immun 1994; 62:769-78.

21 Mills KH, Ryan M, Ryan E, Mahon BP. A murine model in which protection correlates with pertussis vaccine efficacy in children reveals complementary roles for humoral and cell-mediated immunity in protection against Bordetella pertussis. Infect Immun 1997; 66:594-602.

22 Barnard A, Mahon BP, Watkins J, Redhead K, Mills KHG. Th1/Th2 cell dichotomy in acquired immunity to Bordetella pertussis: variables in the in vivo priming and in vitro cytokine detection techniques affect the classification of T cell subsets as Th1, Th2 or Th0. Immunology 1996; 87:372-80.

23 Mahon BP, Sheahan BJ, Griffin F, Murphy G, Mills KHG. Atypical disease after Bordetella pertussis respiratory infection of mice with targeted disruptions of interferon- $\gamma$ receptor or immunoglobulin $\mu$ chain genes. J Exp Med 1997; 186:1843-51.

24 Morokata T, Ishikawa J, Ida K, Yamada T. C57BL/6 mice are more susceptible to antigen-induced pulmonary eosinophilia than $\mathrm{BALB} / \mathrm{c}$ mice, irrespective of systemic $\mathrm{T}$ helper $1 / \mathrm{T}$ helper 2 responses. Immunology 1999; 98:345-51.

25 Strachan D. Hay fever, hygiene and household size. $\mathrm{Br}$ Med $J$ 1989; 299:1259-60.

26 Skerry CM, Cassidy JP, English K, Feunou-Feunou P, Locht C, Mahon BP. A live attenuated Bordetella pertussis candidate vaccine does not cause disseminating infection in IFN- $\gamma$ receptor knockout mice. Clin Vaccine Immunol 2009; 16:1344-51.

27 Grüber C, Warner J, Hill D, Bauchau VEPAAC Study Group. Early atopic disease and early childhood immunization - is there a link? Allergy 2008; 63:1464-72.

28 Martignon G, Oryszczyn MP, Annesi-Maesano I. Does childhood immunization against infectious diseases protect from the development of atopic disease? Pediatr Allergy Immunol 2005; 16:193-200.

29 Peebles RS Jr, Hashimoto K, Collins RD et al. Immune interaction between respiratory syncytial virus infection and allergen sensi- tization critically depends on timing of challenges. J Infect Dis 2001; 184:1374-9.

30 Holgate S, Casale T, Wenzel S, Bousquet J, Deniz Y, Reisner C. The anti-inflammatory effects of omalizumab confirm the central role of IgE in allergic inflammation. J Allergy Clin Immunol 2005; 115:459-65.

31 Hamelmann E, Tadeda K, Oshiba A, Gelfand EW. Role of IgE in the development of allergic airway inflammation and airway hyperresponsiveness - a murine model. Allergy 1999; 54:297-305.

32 Sur S, Wild JS, Choudhury BK, Sur N, Alam R, Klinman DM. Long term prevention of allergic lung inflammation in a mouse model of asthma by $\mathrm{CpG}$ oligodeoxynucleotides. J Immunol 1999; 162:6284-93.

33 Richter A, Puddicombe SM, Lordan JL, Bucchieri F, Wilson SJ. The contribution of interleukin (IL)-4 and IL-13 to the epithelial-mesenchymal trophic unit in asthma. Am J Respir Cell Mol Biol 2001; 25:385-91.

34 Cohn L, Herrick C, Niu N, Homer R, Bottomly K. IL-4 promotes airway eosinophilia by suppressing IFN- $\gamma$ production: defining a novel role for IFN- $\gamma$ in the regulation of allergic airway inflammation. J Immunol 2001; 166:2760-7.

35 Wen FQ, Kohyama T, Liu X et al. Interleukin-4- and interleukin13-enhanced transforming growth factor-beta2 production in cultured human bronchial epithelial cells is attenuated by interferon-gamma. Am J Respir Cell Mol Biol 2002; 26:484-90.

36 Gleich G. Mechanisms of eosinophil-associated inflammation. J Allergy Clin Immunol 2000; 105:651-63.

37 Brusselle GG, Kips JC, Tavernier JH et al. Attenuation of allergic airway inflammation in IL-4 deficient mice. Clin Exp Allergy 1994; 24:73-80.

38 Wills-Karp M, Luyimbazi J, Xu X, Schofield B, Neben TY, Karp CL. Interleukin-13: central mediator of allergic asthma. Science 1998; 282:2258-61.

39 Belcher C, Drenkow J, Kehoe B et al. The transcriptional responses of respiratory epithelial cells to Bordetella pertussis reveal host defensive and pathogen counter-defensive strategies. Proc Natl Acad Sci 2000; 97:13847-52.

40 Das P. Whooping cough makes global comeback. Lancet Infect Dis 2002; 2:322.

41 Hansen G, Berry G, DeKruyff RH, Umetsu DT. Allergen-specific Th1 cells fail to counterbalance Th2 cell-induced airway hyperreactivity but cause severe airway inflammation. J Clin Invest 1999; 103:175-83.

42 Marsland BJ, Scanga CB, Kopf M, Le Gros G. Allergic airway inflammation is exacerbated during acute influenza infection and correlates with increased allergen presentation and recruitment of allergen-specific T-helper type 2 cells. Clin Exp Allergy 2004; 34:1299-306.

43 Gern J. Viral and bacterial infections in the development and progression of asthma. J Allergy Clin Immunol 2000; 105 (Part 2):S497-502.

\section{Supporting Information}

Additional supporting information may be found in the online version of this article: 
Figure S1. H\&tE-stained lung sections. Original magnification $\times 100$. Perivascular and peribronchial inflammation was evaluated as (a) mild, (b) moderate, and (c) severe. (a) Mild peribronchial inflammation (centre) surrounded by clear alveoli and cross-sectioned bronchioles (bottom left, right). (b) Moderate peribronchial inflammation surrounding bronchiole (top right, bottom right) and moderate alveolar inflammation. (c) Severe perivascular and peribronchial inflammation with bronchial epithelial hypertrophy (centre, centre right). Images represent typical pathology at each grade.

Figure S2. BPZE1 challenge prior to OVA sensitisation decreases airway hyperresponsiveness to sensitising allergen. Airway responsiveness was assessed on day 37 by methacholine induced airflow obstruction from conscious mice using whole-body plethysmography in conjunction with the BioSystem XA software (Buxco Electronics, USA) as previously described [16]. (a) Non-sensitised ( $\bullet$ ), OVA-sensitised (- $)$, BPSM infected+OVA sensitisation $(\boxminus)$ and BPZE1 challenge+OVA sensitisation $(\nabla)$. (b) Nonsensitised (Control $\odot$ ), OVA-sensitised (- $)$, non-sensitised BPSM infected $(\diamond)$ and nonsensitised BPZE1 challenge $(\bullet)$. Results are expressed as mean enhanced pause $(\mathrm{PenH}) \pm$ S.E.M. Where no error bars are visible, error bars are shorter than the size of the data point symbol. Data points represent the mean PenH from eight mice in this case.

Please note: Wiley-Blackwell is not responsible for the content or functionality of any supporting materials supplied by the authors. Any queries (other than missing material) should be directed to the corresponding author for the article. 
This document is a scanned copy of a printed document. No warranty is given about the accuracy of the copy. Users should refer to the original published version of the material. 